\title{
BMJ Open What evidence is used to underpin the design of strength-based exercise interventions evaluated in randomised controlled trials for rheumatoid arthritis? A systematic review protocol
}

\author{
Graham Boniface, ${ }^{1}$ Meriel Norris, ${ }^{2}$ Esther Williamson, ${ }^{1}$ Varsha Gandhi, \\ Shona Kirtley, ${ }^{1}$ Neil O'Connell ${ }^{2}$
}

To cite: Boniface G, Norris M, Williamson $\mathrm{E}$, et al. What evidence is used to underpin the design of strengthbased exercise interventions evaluated in randomised controlled trials for rheumatoid arthritis? A systematic review protocol. BMJ Open 2018;8:e24127. doi:10.1136/ bmjopen-2018-024127

- Prepublication history for this paper is available online. To view these files please visit the journal online (http://dx.doi. org/10.1136/bmjopen-2018024127).

Received 11 May 2018 Revised 23 July 2018 Accepted 30 July 2018

\section{Check for updates}

(c) Author(s) (or their employer(s)) 2018. Re-use permitted under CC BY-NC. No commercial re-use. See rights and permissions. Published by BMJ.

${ }^{1}$ Nuffield Department of Orthopaedics, Rheumatology and Musculoskeletal Sciences (NDORMS), University of Oxford, Oxford, UK

${ }^{2}$ Department of Clinical

Sciences, Institute of

Environment, Health and

Societies, Brunel University

London, Uxbridge, UK

Correspondence to

Graham Boniface;

graham.boniface@ndorms.ox. ac.uk

\section{ABSTRACT}

Introduction Healthcare researchers designing strengthbased exercise interventions must choose an appropriate dose to test before evaluating its effect using a definitive/ phase-III randomised controlled trial (RCT). Compared with early phase testing employed by pharmaceutical trials, it is questionable whether exercise-based trials employ the same rigour for establishing tolerated dosage. Consequently, it is unclear if participants are initially prescribed optimal doses of exercise, which may potentially impact on study outcomes. Using trials of strength-based exercise interventions in adults with rheumatoid arthritis (RA) as an exemplar, the aims of this review are to (1) identify the proportion of RCTs that use phase I/II trials with dose escalation methodology for setting prescription parameters, (2) determine type and level of evidence used to justify prescription parameters of strength-based exercise interventions evaluated by RCTs, (3) explore consistency and applicability of the evidence underpinning prescription parameters in RCTs and (4) explore if a relationship exists between risk of bias for RCTs evaluating strength-based interventions and the level of evidence used to underpin prescription parameters. Methods and analysis Focusing on RCT's evaluating strength-based exercise interventions in adults with RA published after 2000, the following databases will be searched: Allied and Complementary Medicine Database, Cochrane Central Register of Controlled Trials, Cumulative Index to Nursing and Allied Health Literature, Excerpta Medica Database, Medline and Physiotherapy Evidence Database. For each RCT, we will identify the evidence used to underpin prescription parameters. Both trial and underpinning evidence will have key information about the intervention extracted using the template for intervention description and replication checklist. Risk of bias will be assessed according to Cochrane. Levels of evidence will be assessed against the 0xford Centre for Evidence-Based Medicine and relationships between RCT and underpinning evidence explored and described narratively. Two independent assessors will be involved throughout data selection and extraction with recourse to a third reviewer should agreement not be reached.

Ethics and dissemination No ethical issues are identified. Dissemination will be via publication.

\section{Strengths and limitations of this study}

- This review presents a novel approach to investigating the dose prescriptions used for exercise interventions evaluated in randomised controlled trials (RCTs) using trials in rheumatoid arthritis as an example.

- It will identify how many RCTs evaluating strengthbased exercise interventions in rheumatoid arthritis report using early phase trials to set prescription parameters.

- This review will examine consistency and applicability of the evidence used by healthcare researchers to underpin the prescription parameters.

- A limitation of the review is that we must rely on the description of interventions provided by the authors, so findings will be reliant on the quality of this reporting.

PROSPER0 registration number CRD42018090963.

\section{INTRODUCTION}

Developing the interventions evaluated as part of a clinical trial is a critical stage of the research process. An integral part of this process focuses on determining safe and effective prescription of dose, yet the approaches used by healthcare researchers differ depending on the type of intervention being tested. Those evaluating investigational medicinal products commonly use early phase clinical trials (eg, phase I/II), employing different dose escalation designs ${ }^{12}$ as an essential step to safeguard participants and optimise potential for efficacy. ${ }^{34}$ Conversely, researchers evaluating complex rehabilitation interventions involving exercise seldom take this approach. Instead, many follow the Medical Research Council (MRC) framework for developing and evaluating randomised 
controlled trials (RCTs) for complex interventions used to improve health. ${ }^{5-7}$ This framework has parallels with the phases used for evaluating investigational medicinal products: (1) preclinical (theory), (2) phase I (modelling), (3) phase II (exploratory trial), (4) phase III (definitive RCT) and (5) phase IV (long-term implementation). Phases 1-3 specifically relate to developing the intervention and include setting prescription parameters (ie, recommended dose and/or schedule).

Eighteen years have passed since the MRC produced the first version of its framework. In both the original (2000) and updated version (2006), the MRC recommends piloting and exploratory clinical testing for addressing key uncertainties (eg, dose parameters). Yet, in a provisional scoping search of the literature, phase I/II trials appear to be poorly adopted by healthcare researchers evaluating exercise-based interventions. However, available studies illustrate that formal investigation of tolerance to an exercise-based intervention in people with health conditions can help to address these uncertainties prior to undertaking a larger, more costly trial. These studies challenge previous research that did not use a dose escalation design. ${ }^{8-10}$ For example, we located two trials which used $3+3$ dose escalation methodology to determine the maximum tolerated dose of exercise interventions. The first involved a multimodal exercise programme (endurance training, progressive resistance training and task-related practice) and its effect on walking endurance, balance and mobility in community dwelling stroke survivors with diminished mobility. ${ }^{11}$ The results indicated that almost double the amount of exercise could be tolerated compared with that used in previous studies in similar cohorts. The second aimed to determine the maximum tolerated dose of walking exercise in people with severe osteoarthritis of the knee. ${ }^{12}$ The maximum tolerated dose of walking was $80 \mathrm{~min}$ less per week than the $150 \mathrm{~min}$ of physical activity recommended by WHO for healthy adults aged $18-64 .^{10}$

In the absence of such information acquired from early phase testing, researchers must rely on expert opinion and/or consensus or draw from the available evidence (eg, previous trials, cohort studies, exercise guidelines for non-clinical populations, etc.) to develop the interventions they wish to evaluate. We hypothesise that the potential impact of this approach is that the exercise interventions being tested in clinical trials are not optimised for specific populations prior to testing. This hypothesis has not been explored previously. Therefore, the aim of this review is to better understand about how healthcare researchers develop exercise interventions and set dose parameters for testing in an RCT.

A large number of exercise interventions have been evaluated across many musculoskeletal conditions and to include all trials of exercise interventions would be beyond the scale of this review. Therefore, we have chosen to focus on trials of strength-based exercise interventions evaluated in patients with rheumatoid arthritis (RA) for this review. RA is a painful and debilitating systemic inflammatory chronic condition ${ }^{13}$ that affects approximately $1 \%$ of the population in the UK. ${ }^{14}$ The economic impact in relation to cost is substantial. ${ }^{15}$ We have chosen strength-based exercise for two reasons: (1) it has been recommended in the management of $\mathrm{RA}^{1617}$ and (2) the optimal dose-response relationships regarding strength training variables (eg, volume, intensity, frequency, etc) remain unclear, ${ }^{18}$ particularly for clinical populations such as RA. ${ }^{19}$

\section{OBJECTIVES}

\section{Primary objective}

1. To determine what proportion of published RCTs evaluating strength-based interventions in RA report using stand-alone phase I/II trials for setting prescription parameters. This includes the authors of the RCTs conducting their own study or specifically citing evidence from such a study.

\section{Secondary objectives}

1. To determine what type and level of evidence is used to underpin prescription parameters of strength-based exercise interventions in RA evaluated by RCTs.

2. Explore the consistency and applicability of the evidence used to underpin between prescription parameters and study populations in RCTs evaluating strengthbased exercise interventions in RA.

3. To explore if a relationship exists between risk of bias for RCTs evaluating strength-based interventions in RA and the level of evidence for underpinning prescription parameters.

\section{METHODS AND ANALYSIS}

\section{Overview of methodological approach}

This protocol was drafted using the Preferred Reporting Items for Systematic Reviews and Meta-Analysis Protocols checklist. ${ }^{20}$ We will identify RCTs (these will be called the primary evidence sources) evaluating strength-based exercise interventions in adults with RA. During data extraction of the primary evidence source, we will identify the citation/s (we will call these the secondary evidence sources) used to underpin the prescribed intervention, specifically the prescription parameters. We will then obtain the secondary evidence source/s and perform further data extraction. Should the secondary evidence source be a literature review, clinical guideline or pilot study, we will identify where possible, the citation/s (these will be called the tertiary evidence sources) most relevant to underpinning the prescription parameters for the intervention from the primary evidence source.

\section{Criteria for considering studies for this review}

We will include all published RCTs where a main component of the intervention being evaluated will be land-based strengthening exercise for adults with RA. Strengthening exercises may involve the individual using equipment (e.g. free weights/machines), or their own bodyweight 
to provide resistance against gravity (e.g. sit-to-stand exercise). The intervention may be unsupervised, supervised or both and carried out individually, or in a group. The strength-based intervention may be multifactorial (eg, used in conjunction with cointerventions like education) or multicomponent (e.g. used with other forms of exercise like flexibility exercise).

\section{Types of participant}

Studies will be eligible if they only include adults (men and women over 18 years of age) with a diagnosis of RA. We will not limit inclusion to studies using classification criteria such as the 2010 American College of Rheumatology/European League Against Rheumatism Classification Criteria for Rheumatoid Arthritis ${ }^{21}$ to ensure we identify as many RCTs using strength-based exercise interventions to treat RA as possible.

\section{Stage 1: search methods for identification of studies \\ Electronic searches}

We will search the following databases: Allied and Complementary Medicine Database (AMED), Cochrane Central Register of Controlled Trials (CENTRAL), Cumulative Index to Nursing and Allied Health Literature (CINAHL), Excerpta Medica Database (Embase), Ovid Medline and Physiotherapy Evidence Database (PEDro) using tailored search strategies. Box 1 presents the search strategy for Medline. We will limit our search to start from April 2000. This is when the MRC published their original framework for developing and evaluating RCTs for complex interventions used to improve health. We present the flow diagram of the study selection process (figure 1).

\section{Searching other resources}

In addition to the above, we will check reference lists of retrieved studies for relevant articles.

\section{Stage 2: selection of studies (title and abstract screening)}

Duplicate records will be removed using the web-based software platform Covidence ${ }^{22}$ commonly used by Cochrane authors for screening and data extraction. Two review authors (GB, VG) will then use this platform to independently screen the title and abstract of each remaining record to determine which studies should be assessed further. We will transfer a record for full-text screening should the review authors disagree.

\section{Stage 3: selection of studies (full-text screening)}

Full text will be uploaded to Covidence where review authors (GB, VG) will investigate all potentially relevant articles. Where full text is not available online, we will contact the corresponding author of the included study to obtain a copy. Disagreement between the review authors (GB, VG) about whether to include or exclude a record will be resolved by discussion. If consensus cannot be reached, we will consult a third reviewer (NO) for arbitration will be implemented. If resolving disagreement is not possible, the article will be added to those 'awaiting assessment' and we will contact the study authors for

\section{Box 1 Medline search strategy}

\section{Search terms}

1. Arthritis, Rheumatoid/

2. (RA or rheumatoid).ti,ab.

3. (rheumatoid adj1 arthritis).ti,ab,kw.

4. or $/ 1-3$

5. Exercise/

6. Exercise Therapy/

7. Plyometric Exercise/

8. Exercise Movement Techniques/

9. Physical Therapy Modalities/

10. Physical Fitness/

11. Physical Endurance/

12. (exercis\$ adj1 (home or programme $\$$ or program $\$$ or therap\$ or technique $\$$ or train\$ or treatment\$ or intervention\$ or supervised)). ti,ab,kw.

13. ((therapeutic or land or intensi\$ or dynamic or isometric or isotonic or isokinetic) adj3 (exercis\$ or train\$)).ti,ab,kw.

14. (physical adj1 (activ\$ or education\$ or fitness or train $\$$ or therap\$ or treatment\$ or intervention\$)).ti,ab,kw.

15. (physio or physiotherap\$).ti,ab,kw.

16. (cycle or cycling or bicycle or walk\$).ti,ab,kw.

17. (physical adj1 condition $\$$ adj1 (exercis\$ or train $\$$ or programme $\$$ or program\$)).ti,ab,kw.

18. ((muscle or grip\$) adj2 (programme\$ or program\$ or therap\$ or technique $\$$ or train $\$$ or treatment\$ or intervention\$ or exercis\$)). $\mathrm{ti}, \mathrm{ab}, \mathrm{kw}$.

19. (hand\$ or wrist\$ or shoulder\$ or knee\$ or ankle\$ or joint\$ or elbow $\$$ or hip or cervical or lumbar or trunk) adj1 (strength $\$$ or exercis\$ or therap\$)).ti,ab,kw.

20. Resistance Training/

21. ((resistance or strength $\$$ or weight or endurance) adj1 (programme $\$$ or program $\$$ or therap $\$$ or technique $\$$ or train $\$$ or treatment\$ or intervention\$ or exercis\$)).ti,ab,kw.

22. $o r / 5-21$

23. 4 and 22

24. randomized controlled trial.pt.

25. controlled clinical trial.pt.

26. randomized.ab.

27. placebo.ab.

28. drug therapy.fs.

29. randomly.ab.

30. trial.ab.

31. groups.ab.

32. or $/ 24-31$

33. 23 and 32

34. exp animals/nothumans.sh.

35. 33 not 34

36. limit 35 to $y r=' 2000-2018$ '

clarification about any points of disagreement raised by the review authors $(\mathrm{GB}, \mathrm{VG}, \mathrm{NO})$ so that a final decision can be made.

\section{Stage 4: data extraction (primary evidence source)}

A data extraction form will be developed and piloted using a representative sample of included studies in order to identify any missing items or unclear coding instructions. Once the data extraction form is finalised, two review authors will independently extract data from 


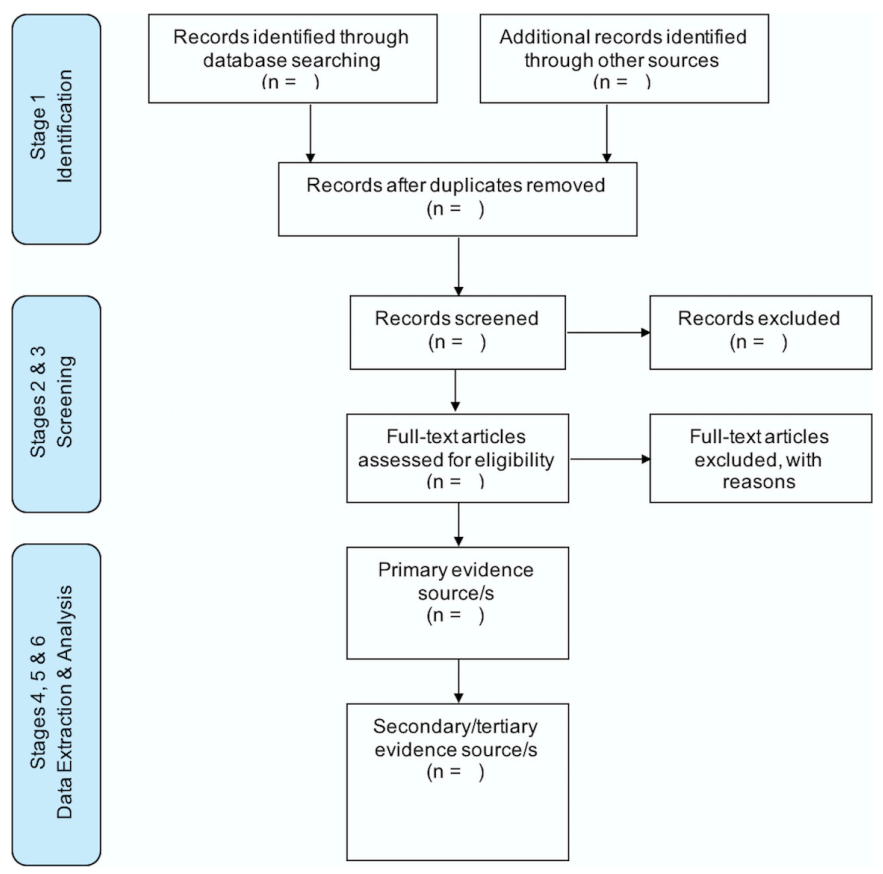

Figure 1 Flow diagram of the study selection process.

the included primary evidence sources. Disagreement will be resolved by discussion. If consensus cannot be achieved, a third review author (NO) will act as arbitrator. The following data will be extracted: 1) trial information (trial design, study location (country), setting, sample size, inclusion and exclusion criteria), (2) participant information (how sample was recruited, study inclusion and exclusion criteria, total number of participants randomised, total number of participants in intervention and control arms, total number of participants lost to follow-up, participant age, participant sex, participant ethnicity), (3) intervention information using the template for intervention description and replication (TIDieR) checklist and guide. ${ }^{23}$ Items 2 and 8 from the TIDieR checklist will assist with identifying whether early phase testing (phase I and II) was undertaken. We will define a phase I-II trial as a stand-alone study that was used by the healthcare researchers of the primary evidence source to learn more about what the best dose to use was and its potential for side effects. Pilot studies conducted within the RCT, whereby the study participants are included in the final analysis, will not be defined as an early phase I-II trial. Where appropriate, we will use intervention and/or protocol publications linked to the primary evidence source to assist with extracting information about the intervention, (4) description of outcomes measures used and brief summary of findings and (5) full citation/s (if used) by the trial authors to underpin the strength-based prescription parameters for the intervention (the secondary evidence source/s). Where appropriate, we will use intervention and protocol publications linked to the primary evidence source to assist with identifying the secondary evidence source/s. We anticipate that some underpinning secondary evidence sources may be a literature review, clinical guideline or pilot study. Should this be the case, we will also identify the tertiary evidence source/s that seem most relevant to underpinning the prescription parameters.

Stage 5: data extraction (secondary/tertiary evidence source) We will obtain full text of the secondary and tertiary evidence source/s identified at stage 4 . Where full text is not available online, we will contact the corresponding author to obtain a copy. We will extract data using the extraction form used in stage 4 . We will indicate when the secondary/tertiary evidence source/s are not primary research.

\section{Stage 6: analysis}

For each primary evidence source, we will outline in table format the information collected at stage 4 . We will then use the question: 'Does this intervention help?' taken from the Oxford Centre for Evidence-Based Medicine-Levels of Evidence ${ }^{24-26}$ to grade the secondary and/or tertiary evidence source/s. Using the information collected with the TIDieR checklist and guide, we will narratively explore and identify areas of homogeneity and heterogeneity between primary evidence source and the underpinning secondary and/or tertiary evidence sources. Inconsistencies in study populations (eg, gender, age, ethnicity, etc), intervention prescription parameters (eg, type of exercise, volume, load, intensity, duration, frequency, etc) and study outcomes (eg, primary and secondary) used will be reported using a simple 'Yes=Consistent', 'No=Inconsistent' or 'Unclear=Not reported' coding strategy. We will also explore narratively the relationship between risk of bias for the primary evidence source and the level of evidence established for the underpinning evidence sources linked to the prescription parameters.

\section{Risk of bias assessment}

For the primary sources, we will assess the risk of bias using the six key domains of the Cochrane risk of bias tool: (1) random sequence generation, (2) allocation concealment, (3) blinding of participants and personnel, (4) blinding of outcome assessors, (5) incomplete outcome data and (6) selective outcome reporting. ${ }^{27}$ Two review authors will independently assess risk of bias of each included RCT. Disagreement will be resolved by discussion, or recourse to a third reviewer (NO). Where appropriate, we will assess risk of bias of the underpinning evidence (secondary and tertiary evidence sources). Where the study is a non-randomised controlled cohort design, we will use Cochrane's Risk Of Bias In Non-Randomized Studies of Interventions tool. ${ }^{28}$

\section{Patient and public involvement}

Patients and the public were not involved in the design of this systematic review.

\section{DISCUSSION}

We present a protocol for a novel systematic literature review to expand our understanding of how healthcare 
researchers develop exercise interventions and set dose parameters for testing in RCTs. This is especially important as the published descriptions of interventions are frequently incomplete. ${ }^{29}$ The use of a narrative approach to analyse the extracted data is essentially exploratory in nature. However, we contend that given the likely mixed and unpredictable nature of the evidence reviewed, such an approach is most appropriate.

Acknowledgements The authors thank Brunel University London, the University of Oxford and the NIHR Collaboration for Leadership in Applied Health Research \& Care 0xford for supporting this review. The views expressed are those of the authors and not necessarily those of the Brunel University London, University of Oxford, the NHS, the NIHR or the Department of Health and Social Care.

Contributors GB devised the study and drafted the protocol and will screen potentially eligible studies, extract data, assess quality and complete data synthesis. NO, MN and EW critically reviewed the protocol manuscript. VG will screen potentially eligible studies, extract data and assess quality. SK contributed to developing and running the search strategies.

Funding This work was supported by the National Institute for Health Research (NIHR) Collaboration for Leadership in Applied Health Research and Care Oxford at Oxford Health NHS Foundation Trust, and supported by the NIHR Biomedical Research Centre, 0xford.

Competing interests None declared.

Patient consent Not required.

Provenance and peer review Not commissioned; externally peer reviewed.

Open access This is an open access article distributed in accordance with the Creative Commons Attribution Non Commercial (CC BY-NC 4.0) license, which permits others to distribute, remix, adapt, build upon this work non-commercially, and license their derivative works on different terms, provided the original work is properly cited, appropriate credit is given, any changes made indicated, and the use is non-commercial. See: http://creativecommons.org/licenses/by-nc/4.0/.

\section{REFERENCES}

1. Hansen AR, Graham DM, Pond GR, et al. Phase 1 trial design: is $3+$ 3 the best? Cancer Control 2014;21:200-8.

2. Le Tourneau C, Lee JJ, Siu LL. Dose escalation methods in phase I cancer clinical trials. J Natl Cancer Inst 2009;101:708-20.

3. Health Research Authority, 2017. Phase 1 clinical trials 2017. https://www.hra.nhs.uk/planning-and-improving-research/policiesstandards-legislation/phase-1-clinical-trials/ (accessed 08 Nov 2017).

4. EMEA. Guideline on strategies to identify and mitigate risks for first-in-human and early clinical trials with investigational medicinal products: EMEA, 2017.

5. MRC. A framework for development and evaluation of RCT's for complex interventions to improve health. London: MRC, 2000.

6. MRC. Developing and evaluating complex interventions: new guidance. London: MRC, 2006.

7. Craig P, Dieppe P, Macintyre S, et al. Developing and evaluating complex interventions: the new Medical Research Council guidance. BMJ 2008;337:a1655.

8. ACSM. Progression models in resistance training for healthy adults. Med Sci in Sports \& Exercise 2009;41:687-708.
9. Garber CE, Blissmer B, Deschenes MR, et al. American College of Sports Medicine position stand. Quantity and quality of exercise for developing and maintaining cardiorespiratory, musculoskeletal, and neuromotor fitness in apparently healthy adults: guidance for prescribing exercise. Med Sci Sports Exerc 2011;43:1334-59.

10. WHO. Global recommendations on physical activity for health. Switzerland: World Health Organisation, 2010.

11. Dite $W$, Langford ZN, Cumming TB, et al. A Phase 1 exercise dose escalation study for stroke survivors with impaired walking. Int $J$ Stroke 2015;10:1051-6.

12. Wallis JA, Webster KE, Levinger $P$, et al. The maximum tolerated dose of walking for people with severe osteoarthritis of the knee: a phase I trial. Osteoarthritis Cartilage 2015;23:1285-93.

13. Suurmeijer TP, Waltz M, Moum T, et al. Quality of life profiles in the first years of rheumatoid arthritis: results from the EURIDISS longitudinal study. Arthritis Rheum 2001;45:111-21.

14. Symmons D, Turner G, Webb R, et al. The prevalence of rheumatoid arthritis in the United Kingdom: new estimates for a new century. Rheumatology 2002;41:793-800.

15. Cooper NJ. Economic burden of rheumatoid arthritis: a systematic review. Rheumatology 2000;39:28-33.

16. NICE. Rheumatoid arthritis in adults: management. Clinical guideline [CG79], 2015.

17. Hurkmans E, van der Giesen FJ, Vliet Vlieland TP, et al. Dynamic exercise programs (aerobic capacity and/or muscle strength training) in patients with rheumatoid arthritis. Cochrane Database Syst Rev 2009;75:CD006853.

18. Borde R, Hortobágyi T, Granacher U. Dose-response relationships of resistance training in healthy old adults: a systematic review and meta-analysis. Sports Med 2015;45:1693-720.

19. Cooney JK, Law RJ, Matschke V, et al. Benefits of exercise in rheumatoid arthritis. J Aging Res 2011;2011:1-14.

20. Moher D, Shamseer L, Clarke M, et al. Preferred reporting items for systematic review and meta-analysis protocols (PRISMA-P) 2015 statement. Syst Rev 2015;4:1-4.

21. Aletaha D, Neogi T, Silman AJ, et al. 2010 Rheumatoid arthritis classification criteria: an American College of Rheumatology/ European League against rheumatism collaborative initiative. Ann Rheum Dis 2010;69:1580-8.

22. Covidence systematic review software, Veritas Health Innovation. Better systematic review management. www.covidence.org

23. Hoffmann TC, Glasziou PP, Boutron I, et al. Better reporting of interventions: template for intervention description and replication (TIDieR) checklist and guide. BMJ 2014;348:g1687.

24. Howick J, Chalmers I, Glsziou P, et al. The 2011 Oxford CEBM levels of evidence (introductory document): Oxford Centre for EvidenceBased Medicine, 2011. (accessed 21 Feb 2018).

25. Howick J, Chalmers I, Glsziou P, et al. Explanation of the 2011 Oxford Centre for Evidence-Based Medicine (OCEBM) levels of evidence (background document). Oxford: Oxford Centre for Evidence-Based Medicine, 2011.

26. OCEBM Levels of Evidence Working Group. The Oxford levels of evidence 2: Oxford Centre for Evidence-Based Medicine, 2016. (accessed 21 Feb 2018).

27. Higgins JP, Altman DG, Gøtzsche PC, et al. The Cochrane Collaboration's tool for assessing risk of bias in randomised trials. BMJ 2011;343:d5928.

28. Sterne JA, Hernán MA, Reeves BC, et al. ROBINS-I: a tool for assessing risk of bias in non-randomised studies of interventions. BMJ 2016;355:i4919.

29. Hoffmann TC, Erueti C, Glasziou PP. Poor description of nonpharmacological interventions: analysis of consecutive sample of randomised trials. BMJ 2013;347:f3755. 